Rix-Lièvre, G., Genebrier, V. (2011). Les interactions joueurs-arbitres au cours d'un match de football : comprendre les altercations ou leur absence. Science \& Motricité, 72, 27-33.

\title{
Les interactions joueurs-arbitres au cours d'un match de football : \\ Comprendre les altercations ou leur absence.
}

\author{
Rix-Lièvre, Géraldine, \\ Clermont Université, Université Blaise Pascal, UFR STAPS, EA 4281, PAEDI, BP 10448, F- \\ 63000 CLERMONT-FERRAND, Geraldine.rix @univ-bpclermont.fr

\section{Genebrier, Vincent,} \\ Etudiant du Master Anthropologie des pratiques corporelles, UFR STAPS, Université Blaise \\ Pascal, vincent.genebrier@etudiant.univ-bpclermont.fr
}

Etude réalisée avec le soutien de La Poste, du conseil régional d'Auvergne et en partenariat avec la Fédération Française de Football

\section{Résumé :}

Ce travail exploratoire questionne l'interaction entre l'arbitre et les joueurs pour comprendre l'émergence ou non d'altercations au cours d'un match de football. L'étude s'intéresse tant aux processus au cours desquels l'arbitre montre et impose quelque chose aux joueurs, qu'à la manière dont le ou les joueurs vivent subjectivement ces moments-là. Trois matchs ont été investigués. Différentes traces d'activités ont été réalisées pour ensuite aider, tour à tour, l'arbitre et quelques joueurs à expliciter leurs vécus. Plusieurs modes de régulation par l'arbitre de son interaction avec les joueurs sont mis en évidence ainsi que la manière dont certains joueurs sont en mesure, tout en vivant des situations à l'écart de ce que l'arbitre impose, de se projeter in fine dans la situation de l'arbitre.

Mots-clés : Anthropologie cognitive, Expérience, Signification incarnée, Autoconfrontation, Régulation. 
Rix-Lièvre, G., Genebrier, V. (2011). Les interactions joueurs-arbitres au cours d'un match de football : comprendre les altercations ou leur absence. Science \& Motricité, 72, 27-33.

\begin{abstract}
Our purpose is to study the interaction between referee and players in order to understand how altercations appear or not during a soccer match. We consider both process whereby referee shows and imposes something to players and how player(s) live(s) subjectively these moments. Three matches have been analyzed. Different traces of activities have been made in order to help referee and players to describe ex post their lived experiences. Several kind of regulation, by referee, of his interaction with player(s) have been highlighted as well as how some players, even if they live situations contrasting with what referee imposes, are able in fine to project themselves in referee's setting.
\end{abstract}

Key-words: Cognitive anthropology, Experience, Embodied signification, Selfconfrontation, Regulation 
Rix-Lièvre, G., Genebrier, V. (2011). Les interactions joueurs-arbitres au cours d'un match de football : comprendre les altercations ou leur absence. Science \& Motricité, 72, 27-33.

Les situations sportives sont des situations d'affrontement où les acteurs du match (arbitres et joueurs, en premier lieu) ont des objectifs différents, voir antagonistes. Pourtant, ils parviennent plus ou moins facilement, avec plus ou moins d'altercations, à interagir. Même si différents travaux soulignent l'existence de comportements agressifs et/ou d'agression envers l'arbitre, la plupart des matchs de football, dans les différents championnats organisés par les instances fédérales, arrivent à leur terme. Nous nous proposons donc de questionner l'interaction entre l'arbitre et les joueurs pour comprendre l'émergence ou non d'altercations voire de comportements violents en empruntant une perspective alternative. L'étude présentée est exploratoire, elle repose sur l'analyse de l'activité de l'arbitre et de quelques joueurs au cours de trois matchs de football.

\section{Des recherches sur les comportements agressifs/transgressifs à l'étude de l'interaction}

La plupart des travaux s'intéressant à l'interaction d'un arbitre à des joueurs envisage cette question sous l'angle des comportements agressifs des uns envers les autres. Deux orientations semblent privilégiées : (1) l'investigation des agressions dont les arbitres peuvent être l'objet, les vécus qui y sont associés et les conséquences sur leur activité d'arbitrage ; (2) l'étude du lien entre des comportements agressifs des joueurs et l'activité de l'arbitre. Après en avoir exposé les principaux résultats, nous explicitons l'émergence d'une perspective alternative pour étudier l'interaction entre l'arbitre et les joueurs au cours d'un match de football.

La première perspective établit l'existence d'agressions physiques, mais surtout verbales des joueurs envers les arbitres (Folkesson, Nyberg, Archer, \& Norlander, 2002). Les travaux s'attachent à mettre en évidence le stress qu'engendre cette éventualité et la possibilité ou non 
Rix-Lièvre, G., Genebrier, V. (2011). Les interactions joueurs-arbitres au cours d'un match de football : comprendre les altercations ou leur absence. Science \& Motricité, 72, 27-33.

d'y faire face en fonction de différentes variables (l'âge, l'expérience, l'optimisme/ pessimisme de l'arbitre...). En effet, des nombreuses recherches montrent que quel que soit le sport, un des facteurs essentiels de stress chez l'arbitre est rapporté à la peur d'être agressé verbalement ou physiquement et/ou à la crainte d'un conflit avec les joueurs (Goldsmith \& Williams, 1992; Kaissidis-Rodafinos, Anshel, \& Sideridis, 1998; Rainey \& Hardy, 1999a, 1999b). Il s'agit aussi de questionner ce qui est vécu comme une agression par les arbitres, notamment en fonction de leur expérience (Phillips, 1985), et ce à quoi ils attribuent la survenue d'un tel comportement chez un joueur par exemple (Fritman, Nyberg, \& Norlander, 2004; Simmons, 2006). Ainsi, les arbitres rapportent ces comportements notamment au manque de connaissance des règles de la part des joueurs, à l'importance de l'enjeu, aux erreurs d'arbitrage, à la volonté d'obtenir de l'arbitre un avantage, au fait que le jeu est un lieu de défoulement ou encore à la nécessité de trouver un responsable à une mauvaise performance (Fritman, et al., 2004).

Si ces travaux concluent que "the occurrence of threat and aggression are not negligeable problems for soccer referees" (Folkesson, et al., 2002, p325) les résultats mentionnent que "63,6\% reported that they had suffered verbal aggression on at least one occasion from soccer players" (Ibid., p322) sur l'ensemble de leur carrière. Ainsi, même s'il ne faut pas passer sous silence les agressions contre les arbitres et qu'il s'agit surtout de les combattre, d'autres recherches paraissent nécessaire pour :

-établir la fréquence de ces agressions : les chiffres de ces trois dernières années montrent que des agressions physiques contre les arbitres sont constatées dans moins de $0,75 \%$ des matchs des championnats français amateurs (Wincke, 2010),

-identifier les interactions dans lesquelles ce type de comportement apparait et étudier leurs dynamiques. 
Rix-Lièvre, G., Genebrier, V. (2011). Les interactions joueurs-arbitres au cours d'un match de football : comprendre les altercations ou leur absence. Science \& Motricité, 72, 27-33.

Sur ce second point, le travail de Friman et al (2004) met l'accent sur les facteurs/raisons que l'arbitre mobilise pour expliquer a posteriori la survenue d'un incident. Il serait alors intéressant d'examiner si en situation de match, au cours de son interaction avec les joueurs, ce sont ces éléments qui sont prégnants pour anticiper et/ou faire face à ce type de comportement de la part des joueurs; autrement dit si ces éléments permettent ou non de comprendre la dynamique d'une interaction où survient une agression contre l'arbitre.

La seconde perspective questionne la part de l'activité de l'arbitre dans l'apparition, chez les joueurs, de comportements agressifs. L'impact émotionnel des erreurs d'arbitrage et ses implications possibles en termes de comportements agressifs du joueur envers l'arbitre et/ou envers ses adversaires sont notamment étudiés (Reynes, Canovas, Ferrand, \& Pantaleon, 2008). Il apparait ainsi que des erreurs d'arbitrage sont génératrices de sentiments d'énervement, de colère et d'injustice d'autant plus qu'elles se répètent. Distinguant deux catégories d'erreurs d'arbitrage : faux positif (une faute sifflée alors qu'il n'y a pas de faute), faux négatif (une faute est commise mais elle n'est pas sifflée), Reynes, et al. (2008) montrent que selon le type d'erreur et le fait qu'elle se répète ou non, les réactions sont plus ou moins agressives, de natures différentes et n'ont pas la même cible. Les comportements agressifs envers l'arbitre sont plutôt de nature verbale et paraissent survenir plutôt en cas de faux positifs répétés. Les agressions physiques, quant à elles, semblent être généralement dirigées vers les joueurs, notamment en cas de faux négatif. Autrement dit, le fait que l'arbitre ne sanctionne pas une faute, d'autant plus qu'il y a atteinte à l'intégrité physique, peut entrainer des altercations et/ou agressions des joueurs entre eux.

"Cependant, il semble que certains facteurs connexes soient de nature à moduler cette émotion [celle des joueurs victimes d'un faux négatif] en l'accentuant ou en l'atténuant" (Reynes et al, 2008, p9) notamment l'attitude de l'adversaire, l'historique de l'opposition ou 
Rix-Lièvre, G., Genebrier, V. (2011). Les interactions joueurs-arbitres au cours d'un match de football : comprendre les altercations ou leur absence. Science \& Motricité, 72, 27-33.

l'utilité de l'action. Il s'agit alors de relativiser la portée des décisions de l'arbitre et de son interaction avec les joueurs dans l'apparition de comportements agressifs au sein d'une rencontre. Afin de saisir l'impact émotionnel des décisions de l'arbitre et leur portée dans la dynamique de l'interaction, il serait aussi intéressant d'étudier quand et comment en situation de match, les joueurs perçoivent une erreur d'arbitrage. En effet, au cours d'une interaction sportive, les événements ne se donnent pas d'emblée comme tel, ce qui est le cas dans la situation expérimentale proposée.

Partant non plus de constats de violences au sein du football et/ou sur les terrains de sport, mais d'un travail sur l'activité de l'arbitre en match (Rix, 2005, 2007), la perspective que nous proposons aborde les questions d'interaction de manière décalée tout en proposant des prolongements aux études précédentes. Nos recherches aboutissent à une conception alternative de l'arbitrage : l'arbitre n'est plus envisagé comme celui qui doit faire appliquer la règle et/ou gérer les comportements transgressifs, mais comme un acteur qui co-construit avec les joueurs le déroulement du jeu (Rix, Ibid.).

En effet, si l'on considère l'acte de juger relativement à ce qu'il produit, l'arbitre, tout au long du match, montre et impose aux joueurs ce qui est possible dans le cadre de la rencontre. Ainsi, il impose à tous, dans un moment particulier, sa propre manière d'appréhender et de décrire l'activité des joueurs : comme un fait, au regard de sa dynamique ou comme une situation problématique (Rix, 2005). Ce qu'il impose -et la manière dont ceci se construitn'est pas le produit du calcul d'une solution optimale en fonction d'un contexte donné, mais de l'ordre d'une rationalité en acte. Cette dernière reste largement implicite, syncrétique et pré-réfléchie : elle ne se manifeste que corporellement dans, par et au cours de son acte de jugement. Elle est envisagée comme une signification incarnée, spontanée et située qui se réalise et se détermine dans un accomplissement corporel qui montre et impose aux joueurs ce 
Rix-Lièvre, G., Genebrier, V. (2011). Les interactions joueurs-arbitres au cours d'un match de football : comprendre les altercations ou leur absence. Science \& Motricité, 72, 27-33.

qui est possible. Ce faisant, nous considérons que l'arbitre co-construit avec les joueurs le déroulement du jeu. Au regard de ce résultat, c'est la question de l'interaction de l'arbitre avec les joueurs qui se pose et celle de sa dynamique pour comprendre l'émergence ou non d'altercations.

Si, pour étudier l'activité de l'arbitre, nous avons dû saisir la signification qu'il confère spontanément à l'activité des joueurs, c'est-à-dire le processus au cours duquel il leur montre et leur impose quelque chose, travailler sur les processus sous-jacents à la dynamique de l'interaction suppose d'approcher aussi la manière dont le ou les joueurs vivent subjectivement ce moment-là. Il s'agit non plus de caractériser le comportement d'un joueur comme agressif/transgressif et d'en interroger les causes, mais d'investiguer le vécu de chacun et la manière dont il construit sa situation et ce faisant d'éclairer la dynamique de l'interaction entre l'arbitre et les joueurs au cours de matchs de football. Dans la mesure une observation ne paraît pas suffisante pour approcher ce que vit subjectivement un acteur à un moment donné, il s'agit d'adopter une méthode qui permette d'aider chacun à dire les significations qu'il confère spontanément à un contexte dans un moment particulier. Différentes méthodes se sont données cet objectif (Rix \& Biache, 2004; Theureau, 1992; Vermersch, 1994). Dans cette recherche, nous avons opté pour différents entretiens utilisant des enregistrements vidéo comme support. En effet, pour saisir ce qu'un acteur a effectivement vécu dans un moment particulier, l'utilisation d'une vidéo comme trace d'activité présente deux avantages: "(1) spécifier le moment dont il est question, (2) constituer une trace dynamique du déroulement de la pratique" (Rix-Lièvre, 2010, p366). Ainsi, pour approcher ce qui fait sens pour un arbitre tout au long du match, nous l'avons invité à expliciter le décours de son activité à partir d'un enregistrement de sa perspective subjective située, c'est-à-dire d'un point de vue physique proche du sien en situation ; menant ainsi avec l'arbitre, un entretien en re situ subjectif (Rix \& Biache, 2004). Pour solliciter les 
Rix-Lièvre, G., Genebrier, V. (2011). Les interactions joueurs-arbitres au cours d'un match de football : comprendre les altercations ou leur absence. Science \& Motricité, 72, 27-33.

joueurs, le dispositif d'auto-confrontation (Theureau, 1992) a été retenu : c'est à partir d'un enregistrement du match d'un point de vue extérieur, des tribunes et en plan large, que les joueurs ont été invités à expliciter leurs vécus dans des moments particuliers.

\section{Méthode :}

Participants : Ce travail est une étude exploratoire qui repose sur l'analyse de l'interaction entre l'arbitre et les joueurs au cours de trois matchs de CFA/CFA2. Trois arbitres différents ont participé. Cinq clubs ont aussi contribué à ce travail, neufs joueurs ont été plus particulièrement sollicités.

\section{Construction des matériaux :}

Les choix des rencontres investiguées ont été effectués conjointement entre l'équipe de recherche et les instances nationales de l'arbitrage de la Fédération Française de Football. Une fois le match identifié, le chercheur prenait soin de contacter l'arbitre et les clubs concernés par la rencontre afin de présenter la recherche effectuée, son positionnement et ses objectifs, et de solliciter la participation de chacun au travail.

Lors de chaque rencontre, différentes traces de l'activité de l'arbitre et des joueurs ont été construites. Deux enregistrements vidéo ont été réalisés : (1) un enregistrement du match en plan large à partir des tribunes qui constitue une trace des comportements de l'arbitre et des joueurs, (2) un enregistrement audio-vidéo d'une perspective proche de celle de l'arbitre en situation, perspective dite subjective située, construite en équipant l'arbitre d'une caméra (Ø 8mm) fixée au niveau de sa tempe et d'un système d'enregistrement embarqué (3 boitiers de respectivement $8 \times 5 \times 2 \mathrm{~cm}, 9 \times 5 \times 1,5 \mathrm{~cm}, 7,5 \times 6,5 \times 2 \mathrm{~cm}$, pesant au total $350 \mathrm{~g}$ ) placé dans une ceinture identique à celle utilisée pour porter les appareils de transmission audio en Ligue 1 . 
Rix-Lièvre, G., Genebrier, V. (2011). Les interactions joueurs-arbitres au cours d'un match de football : comprendre les altercations ou leur absence. Science \& Motricité, 72, 27-33.

Dans la semaine suivant chaque match, différents entretiens ont été réalisés : (1) un entretien en re situ subjectif avec l'arbitre visant à saisir son vécu tout au long du match, (2) des entretiens d'auto-confrontation avec 2 à 4 joueurs pour là aussi approcher ce qui importe pour eux dans quelques moments du match identifiés en amont par le chercheur. Les joueurs et les moments ont été sélectionnés par le chercheur en fonction des échanges patents avec l'arbitre, mais aussi en fonction de leurs statuts.

\section{Traitement des matériaux :}

Les entretiens ont tout d'abord été intégralement retranscrits. Puis, ont été repérées les verbalisations ayant trait au vécu tant dans les entretiens des arbitres que ceux des joueurs. Deux types de traitement ont ensuite été développés. Les entretiens des arbitres ont fait l'objet d'un traitement longitudinal pour étudier les processus de régulation à l'œuvre tout au long du match. Les moments pour lesquels nous disposions de verbalisations tant du vécu de l'arbitre que d'un ou plusieurs joueurs ont été traités de manière spécifique. Nous avons mis en parallèle une description du contexte, les verbalisations de l'arbitre et celles des joueurs afin de questionner le caractère partagé ou disjoint de leurs situations et son évolution au cours même de l'interaction.

\section{Résultats :}

Les résultats que nous proposons sont issus du traitement des matériaux de l'un des trois matchs investigués. Ils ne sont en aucun cas généralisables en l'état mais permettent d'envisager les perspectives les plus pertinentes dans la poursuite de ce travail.

Certains éléments issus des entretiens avec les arbitres et/ou les joueurs permettent en premier lieu de discuter les résultats issus des travaux sur les comportements agressifs. Par exemple, cet extrait d'entretien laisse supposer que les facteurs évoqués par les arbitres pour expliquer 
Rix-Lièvre, G., Genebrier, V. (2011). Les interactions joueurs-arbitres au cours d'un match de football : comprendre les altercations ou leur absence. Science \& Motricité, 72, 27-33.

a posteriori le comportement des joueurs (Friman et al., 2004) ne sont pas nécessairement ceux qui président à ce qui importe pour lui en situation.

«A : Là pourquoi le capitaine il vient râler? Car il essaie de m'influencer, c'est vrai, ils avaient pas fait beaucoup de fautes et c'était les rouges qui avaient fait plus de petites fautes en première mi-temps et c'est vrai que pour l'ensemble du truc, les rouges auraient mérité d'avoir un avertissement avant, mais y'avait pas... c'est que des petites fautes quoi! Mais là c'est vraiment une faute, une grosse faute et malheureusement c'est un jaune qui a fait la faute! [...]

C : Et t'as pensé à tout ça avant de le mettre le carton?

A : Et... non j’ai pas pensé à tout ça, non. Parce que j’étais tellement pris par la faute »

Mais au-delà du non recouvrement, largement établi, entre les facteurs explicatifs évoqués dans les discours et les significations à l'œuvre dans l'action (Récopé, Lièvre, \& Rix-Lièvre, 2010 ; Rix-Lièvre, 2010), ce travail permet d'envisager la régulation par l'arbitre de son interaction avec les joueurs et des joueurs entre eux. En effet, l'analyse des verbalisations de l'arbitre relatives à son vécu mettent en évidence que si ses actes de jugements imposent aux joueurs une manière de jouer au football -donc une manière d'interagir entre eux pendant que le jeu se déroule-, ils peuvent aussi imposer la déférence (Goffman, 1974) que les joueurs doivent lui témoigner.

«Donc le 10 là, il a eu un coup sur la tête et le capitaine dit «oh rien du tout je le touche pas » et il part. Donc je lui dis... et pourquoi je le fais venir là ? Parce que je l'aurais pas fait venir... mais c'est parce qu'il m'a dit «non y'a rien, je l'ai pas touché ». Et moi je me suis dit, il faut que je le fasse venir pour pas laisser passer ça! Parce que il me prend pour euh... il se fiche de moi un peu quoi en partant et 
Rix-Lièvre, G., Genebrier, V. (2011). Les interactions joueurs-arbitres au cours d'un match de football : comprendre les altercations ou leur absence. Science \& Motricité, 72, 27-33.

tout, donc c'est pour ça que je lui dis de revenir. Au moins il a compris que voilà, que c'était comme ça! Pour moi il y avait quelque chose. »

Le fait d'imposer au joueur de revenir au moment où le jeu est arrêté constitue ici une manière de réguler les rîtes d'interaction (Goffman, 1974), c'est-à-dire de faire exister des règles d'interaction entre l'arbitre et les joueurs, de les construire implicitement et progressivement et d'affirmer le rôle que l'arbitre entend jouer. Mais cette régulation opère aussi au cours même du jeu. Tout en imposant la manière dont le jeu peut se dérouler, l'arbitre circonscrit les interactions possibles par la suite.

Là «jouez, jouez », parce que j'estime qu'il n'y a pas faute et qu'il se relève pas et qu'il réclame pas la faute quoi!

Ce type de régulation peut être identifié notamment lors d'acte de jugement (Rix, 2005) qui imposent que le jeu se poursuit. Le jeu pourrait se poursuivre si l'arbitre n'indiquait rien, mais le fait d'imposer «Jouez, jouez » implique d'une part que le jeu se poursuit, d'autre part réduit le champ d'interactions possibles par la suite. En effet, ces injonctions lèvent l'incertitude des événements : les actions et interactions entre les joueurs sont acceptables dans le cadre d'un match de football, mais surtout le font exister pour les joueurs. Elles matérialisent le fait que l'arbitre a jugé et tendent à réduire les contestations futures c'est-àdire circonscrire les interactions possibles avec l'arbitre non plus seulement sur la forme mais sur le fond. Au regard des résultats de Reynes et al (2008), il faudrait envisager si ces régulations pourraient éviter/réduire les faux négatifs perçus par les joueurs en match et ainsi les agressions physiques entre eux. Ces dernières semblent aussi pouvoir être atténuées dans le cours du jeu par une présence contraignante de l'arbitre auprès des joueurs.

Et donc là je dis «sans les mains, sans les mains » parce que souvent quand... dans la surface, près des buts, y'a souvent du tirage de maillot et tout ça! Donc j'anticipe pour que celui qui voudra prendre la main ou tirer, pour qu'il 
Rix-Lièvre, G., Genebrier, V. (2011). Les interactions joueurs-arbitres au cours d'un match de football : comprendre les altercations ou leur absence. Science \& Motricité, 72, 27-33.

m'entende et qu'il se dise "ah ça se voit qu'il est en train de regarder »! Alors que c'est pas forcément que je le vois le gars! Mais ça évite qu'il le fasse quoi!

Ce que dit l'arbitre impose aux joueurs tant une manière de jouer au football que sa propre présence qui renforce le contrôle et la régulation de l'interaction sportive entre les joueurs.

Ainsi, si aucun conflit n'a été observé au cours de ce match, notre étude permet d'observer des mécanismes de régulation qui semblent autant de processus permettant d'éviter l'émergence d'altercations et/ou de conflits. Dans une perspective de lutte contre les violences sur les terrains de football, il pourrait être intéressant de mettre à jour les différents mécanismes -largement implicites- de régulations que développent les arbitres expérimentés afin de les intégrer au plus tôt dans la formation des arbitres. Ceci permettrait peut-être d'atténuer le fait que "the younger referees were most exposed (and may have been most vulnerable) to threat and aggression » (Folkesson et al., 2002, p325).

Le travail effectué mettant en parallèle les verbalisations relatives aux vécus des joueurs et de l'arbitre a ouvert un questionnement sur le caractère partagé ou disjoint des situations de l'arbitre et des joueurs et sur leurs inscriptions dans le décours de l'interaction. Les matériaux que nous avons construits ne nous permettent de prendre en charge cette question que sur des fautes effectives sifflées par l'arbitre. Le premier apport de ce second traitement est de mettre en évidence que malgré l'absence de conflit patent, les joueurs construisent quelques fois une situation très à l'écart de ce que l'arbitre impose sans pour autant manifester de contestation.

\section{Insérer Tableau 1 : Parallèle entre les situations vécues par l'arbitre et par un joueur}

Ce qui importe à ce moment là pour le joueur, c'est le fait de ne pas avoir touché son adversaire, pour l'arbitre, c'est une action impressionnante qui est perçue dans une dynamique : «je le vois venir, je vois qu'il va faire faute ». Ainsi, les deux situations, celle de l'arbitre et celle du joueur, apparaissent disjointes tant relativement à ce qui est significatif 
Rix-Lièvre, G., Genebrier, V. (2011). Les interactions joueurs-arbitres au cours d'un match de football : comprendre les altercations ou leur absence. Science \& Motricité, 72, 27-33.

(«je l'ai pas touché » versus «c'est par derrière ») qu'à la manière dont ce qui est significatif est construit (fait versus dynamique d'une action) sans pour autant qu'une altercation/un conflit entre le joueur et l'arbitre n'émerge. Le cadre théorique proposé par Reynes et al (2008), emprunté à Weber, parait ici intéressant pour interpréter l'absence de contestation malgré des situations disjointes : le joueur «subit une décision émanant d'un tiers avec lequel il est dans une relation hiérarchique dissymétrique défavorable et face à laquelle il n'a aucun recours» (Ibid., p11). Ainsi, la position de pouvoir de l'arbitre par rapport au joueur (Rix, 2005) serait intégrée et le joueur ne chercherait pas à contester malgré «l'injustice perçue, et des sentiments de dépit/résignation » (Reynes et al., 2008, p11). Pourtant, si l'énervement selon la terminologie du joueur est présent, ce qui apparait relève plus d'une projection de la situation que l'arbitre a pu construire que d'une résignation à une autorité arbitraire : $C$ : Mais t'as pas essayé de protester? 3R: Ben, non, parce qu'après c'est un tacle par derrière aussi... le seul truc c'est qu'au fond de moi je le touche pas, après il siffle... ». Dans un autre cas où un autre joueur construit une situation à l'écart de celle imposée par l'arbitre, un nouveau facteur apparait : ne pas « agacer l'arbitre ».

«Ouais pas l'agacer [l'arbitre], parce que je me dis peut-être qu'à un moment donné, il va le voir que je râle pas, que je me prends des coups et que je me tais, et peut-être à un moment donné, si j'ai une petite faute sur moi dans la surface ou à droite, à gauche, ben il va siffler parce qu'il se dit «bon il doit y avoir faute». Alors que le mec qui est chiant, qui arrête pas, qui demande, qui râle, ben y'aura une petite faute sur lui, même si elle est petite, ben il sifflera pas! Il sifflera pas parce que le mec il demande tout le temps, il est chiant! Alors que le mec qui demande pas trop, là il peut siffler! Et c'est souvent comme ça! C'est logique, c'est humain!» 
Rix-Lièvre, G., Genebrier, V. (2011). Les interactions joueurs-arbitres au cours d'un match de football : comprendre les altercations ou leur absence. Science \& Motricité, 72, 27-33.

Si cet extrait met en évidence ce nouveau facteur, il montre aussi que le joueur construit la manière dont l'arbitre peut percevoir son comportement. Dans les deux cas, les joueurs mobilisent des connaissances expérientielles qui leur permettent de se projeter dans la situation de l'arbitre et d'agir selon ce que ce dernier impose sans incident malgré une situation vécue au départ disjointe de celle imposée par l'arbitre. Ces connaissances qui permettent de saisir/construire ce que l'autre pense, la logique qui préside à son action et la manière dont il peut agir, semblent importantes pour le bon déroulement de l'interaction. Dans la mesure où elles sont plus ou moins implicites, construites dans et par l'expérience, une manière de lutter contre les comportements déviants sur les terrains de football pourrait être de proposer des activités en commun entre les arbitres et les joueurs et/ou de mêler la pratique du jeu à celle de l'arbitrage. Des initiatives comme «Sois foot, joue dans les règles » (Bourdin, Ducloz, 2010) participent de cette démarche.

L'étude de cas présentée, centrée sur la compréhension des processus sous-jacents aux interactions joueurs-arbitres, a permis de mettre en évidence différents mécanismes de régulation, par l'arbitre, de l'interaction. Imposer la déférence que les joueurs doivent lui témoigner, circonscrire le contenu possible des interactions futures, affirmer une présence contraignante paraissent autant de processus contribuant à la construction progressive de modalités partagées d'interaction. Afin de saisir comment ces derniers participent effectivement du bon déroulement de l'interaction, il s'agit d'effectuer un travail de mise en parallèle des situations vécues sur des moments où l'arbitre impose quelque chose aux joueurs sans que ceci ne se traduise nécessairement par une sanction. Cependant, cette première étude montre déjà l'intérêt pour comprendre l'émergence ou non de conflit de s'intéresser aux vécus des joueurs et de l'arbitre tout au long du match, et notamment aux possibilités des uns et des 
Rix-Lièvre, G., Genebrier, V. (2011). Les interactions joueurs-arbitres au cours d'un match de football : comprendre les altercations ou leur absence. Science \& Motricité, 72, 27-33.

autres de saisir/construire ce que l'autre pense, la logique qui préside à son action et la manière dont il peut agir.

\section{Bibliographie :}

Bourdin, N. \& Ducloz, L. (2010). « Sois foot, joue dans les règles » : un exemple concret d'éducation par l'apprentissage de règles de jeux et de vie au travers de la pratique du football. Communication au Colloque SHERPAS « De la violence des terrains au terrain des violences : regards croisés sur le football amateur », Lieven, 27-28 Mai.

Folkesson, P., Nyberg, C., Archer, T., \& Norlander, T. (2002). Soccer referees' experience of threat and agression: effect of age, experience and life orientation on outcom of coping strategy. Agressive behavior, 28, 317-327.

Fritman, M., Nyberg, C., \& Norlander, T. (2004). Threats and Agression directed at soccer referees: an empirical psychological study. The Qualitative Report, 9(4), 652-672.

Goffman, E. (1974). Les rîtes d'interaction. Paris: Edition de minuit.

Goldsmith, P. A., \& Williams, J. M. (1992). Perceived Stressors for Football and Volleyball Officials from Three Rating Levels. Journal of Sport Behavior, 15(2), 106-118.

Kaissidis-Rodafinos, A., Anshel, M. H., \& Sideridis, G. (1998). Sources, Intensity, and Responses to Stress in Greek and Australian Basketball Referees. International Journal of Sport Psychology, 29(4), 303-323.

Phillips, C. L. (1985). Sport group behavior and officials' perceptions. Journal of sport psychology, 16, 1-11.

Rainey, D. W., \& Hardy, L. (1999a). Assaults on Rugby Union Referees : A Three Union Survey. Journal of Sport Behavior, 22, 105-113. 
Rix-Lièvre, G., Genebrier, V. (2011). Les interactions joueurs-arbitres au cours d'un match de football : comprendre les altercations ou leur absence. Science \& Motricité, 72, 27-33.

Rainey, D. W., \& Hardy, L. (1999b). Sources of stress, burnout, and intention to terminate among rugby union referees. Journal of sport sciences, 17, 797-806

Récopé, M., Lièvre, P., \& Rix-Lièvre, G. (2010). The commitment of poalr expedition members to a project: declared motivation or in situ mobilization. Project Management Journal, 41(3), 45-56.

Reynes, E., Canovas, S., Ferrand, C., \& Pantaleon, N. (2008). Conséquences émotionnelles des erreurs d'arbitrage chez les footballeurs: étude exploratoire. Psicologia \& Sociedade, $20(1), 5-15$.

Rix, G. (2005). Typologie des actes de jugement de l'arbitre de rugby expérimenté. Science et Motricité, 56(3), 109-124.

Rix, G. (2007). Le 31ème homme : un garant de la culture du rugby à XV. In J.-Y. Guillain \& P. Porte (Eds.), La planète rugby. Regards croisés sur l'ovalie (pp. 331-350). Paris: Musée National du Sport et Atlantica.

Rix, G., \& Biache, M.-J. (2004). Enregistrement en perspective subjective située et entretien en re situ subjectif : une méthodologie de constitution de l'expérience. Intellectica, 38, 363-396.

Rix-Lièvre, G. (2010). Différents modes de confrontation à des traces de sa propre activité. Entre convergences et spécificités. Revue d'anthropologie des connaissances, 4(2010/2), $357-376$.

Simmons, P. (2006). Tackling Abuse of Officials: Attitudes and Communication Skills of Experienced Football Referees. Paper presented at the ANZCA, "Empowerment, Creativity and Innovation: Challenging Media and Communication in the 21st Century”, The University of Adelaide, Australia, http://www.adelaide.edu.au/anzca2006/conf_proceedings/simmons_peter_tackling_abuse $\underline{\text { football_referees.pdf }}$ 
Rix-Lièvre, G., Genebrier, V. (2011). Les interactions joueurs-arbitres au cours d'un match de football : comprendre les altercations ou leur absence. Science \& Motricité, 72, 27-33.

Theureau, J. (1992). Le cours d'action, analyse sémiologique : essais d'une anthropologie cognitive située. Berne: Peter Lang.

Vermersch, P. (1994). L'entretien d'explicitation. Paris: ESF.

Wincke, P. (2010). Observatoire des comportements. Conférence au Colloque SHERPAS «De la violence des terrains au terrain des violences : regards croisés sur le football amateur », Lieven, 27-28 Mai. 


\begin{tabular}{|c|c|c|}
\hline Description du contexte & Verbalisations du 3R & Verbalisations de l'arbitre \\
\hline 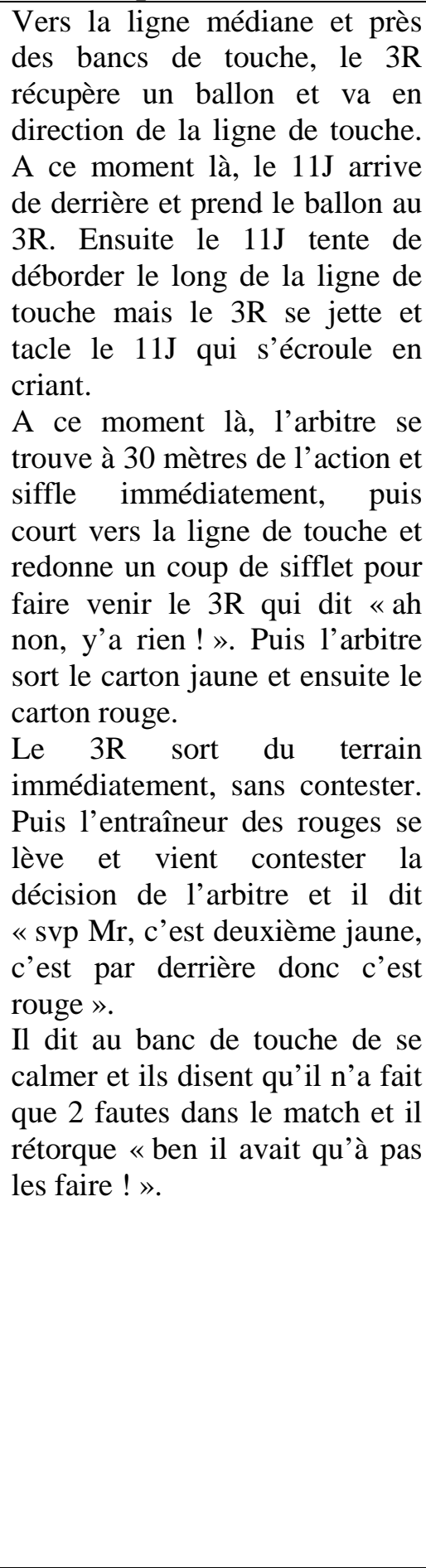 & $\begin{array}{l}3 R \text { : Là alors quand je le tacle, } \\
\text { franchement je joue le ballon et } \\
\text { je pensais vraiment, alors } \\
\text { encore pire que la première... là } \\
\text { je pensais vraiment l'avoir. } \\
\text { Remets la vidéo tu vas voir. } \\
\text { Parce que le ballon est là, là tu } \\
\text { vois quand je tacle je pense } \\
\text { vraiment l'avoir. } \\
C: \text { Tu regardes quoi là ? Que le } \\
\text { ballon? } \\
3 R \text { : Ah oui que le ballon ! Là } \\
\text { j’y vais vraiment, vraiment pas } \\
\text { pour le prendre. Là je veux } \\
\text { vraiment prendre le ballon. [...] } \\
3 R: \text { Ah mais là je lui dis que je } \\
\text { le touche pas! Il me met le } 2 \text { eme } \\
\text { jaune, alors là j'étais énervé. } \\
\text { Parce que je sais qu'au fond de } \\
\text { moi, je le touche pas. } \\
C: \text { Mais t'as pas essayé de } \\
\text { protester? } \\
3 R \text { : Ben , non, parce qu'après } \\
\text { c'est un tacle par derrière } \\
\text { aussi...le seul truc c'est qu'au } \\
\text { fond de moi je le touche pas, } \\
\text { après il siffle... [...] } \\
3 R \text { : Ben je prends le rouge } \\
\text { et...je le touche pas mais au } \\
\text { fond de moi je me dis, ouais } \\
\text { c'est peut-être par derrière... } \\
\text { Mais tu vois il tombe mais sur } \\
\text { le moment je me dis pas « ouais } \\
\text { c'est bien joué de sa part ». } \\
\text { Mais je me dis tu vois y'a le } \\
\text { tacle par derrière. Et là il me } \\
\text { met le rouge, machin et là } \\
\text { j'étais plus dégouté que rageur! } \\
\text { J'avais pas la rage de lui dire } \\
\text { «ouais je le touche pas », } \\
\text { j'étais écœuré. J'étais dégouté } \\
\text { ouais. Mais moi je comprends } \\
\text { pas... }\end{array}$ & $\begin{array}{l}\text { A : Je cours pour y aller parce } \\
\text { que ils étaient limites extérieurs, } \\
\text { ils étaient tous les deux à } \\
\text { l'extérieur parce qu'ils s'étaient } \\
\text { emportés. Et je dis « oh purée » } \\
\text { car la faute est impressionnante } \\
\text { aussi donc j'y vais pour le jaune } \\
\text { et je dis « oula » et avec la } \\
\text { lumière du truc je voyais pas } \\
\text { qui c'était et je me dis «c'est le } \\
\text { 3, c'est le } 3 \text {, il a déjà jaune, il a } \\
\text { déjà jaune » et puis ben j'étais } \\
\text { parti pour mettre jaune à } \\
\text { n'importe quel joueur donc } \\
\text { j'allais pas changer... } \\
\text { [...] Comme la première pour le } \\
7, \text { exactement pareil, et je le } \\
\text { vois venir, je vois qu'il va faire } \\
\text { faute et surtout que... par contre } \\
\text { j'étais pas focalisée sur le } \\
\text { numéro, je savais pas mais en } \\
\text { courant je me dis c'est } \\
\text { dommage il a déjà un jaune. } \\
C: \text { T'as vu que la faute elle } \\
\text { était importante? } \\
\text { A : Ouais elle méritait ! [...] } \\
\text { Je suis tellement...je suis } \\
\text { focalisé sur le joueur... et en } \\
\text { m'approchant j'essaye de } \\
\text { bien...de...parce qu'il était } \\
\text { tourné et puis y'avait la tribune } \\
\text { donc c'était sombre et j' avais } \\
\text { l'impression que c'était le } 3 \\
\text { mais je savais pas et en me } \\
\text { rapprochant j'ai vu que c'était } \\
\text { lui ! Mais non là tu penses pas, } \\
\text { t'es tellement... parce que pour } \\
\text { moi c'était tellement évident } \\
\text { que ça méritait un jaune... }\end{array}$ \\
\hline
\end{tabular}

Tableau 1 : Parallèle entre les situations vécues par l'arbitre et par un joueur 